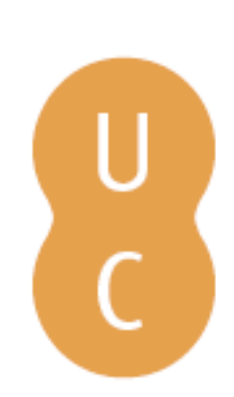

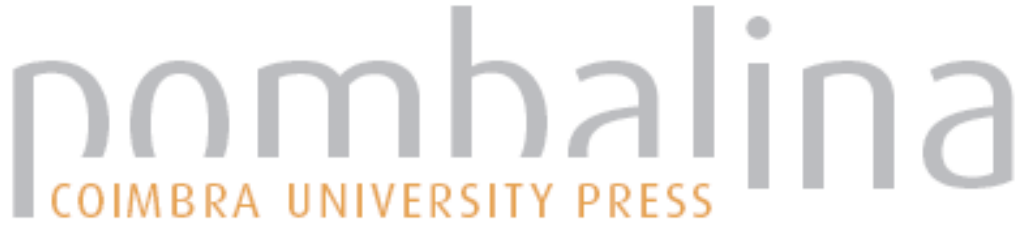

\section{Inventário do Luto para os Cuidadores de Marwit-Meuser - Forma Reduzida (MMCGI-} SF)

Autor(es): $\quad$ Areia, Neide; Major, Sofia; Relvas, Ana Paula

Publicado por: Imprensa da Universidade de Coimbra

URL

persistente: URI:http://hdl.handle.net/10316.2/40590

DOI: $\quad$ DOI:https://doi.org/10.14195/978-989-26-1268-3_6

Accessed : $\quad$ 26-Apr-2023 13:19:31

A navegação consulta e descarregamento dos títulos inseridos nas Bibliotecas Digitais UC Digitalis, UC Pombalina e UC Impactum, pressupõem a aceitação plena e sem reservas dos Termos e Condições de Uso destas Bibliotecas Digitais, disponíveis em https://digitalis.uc.pt/pt-pt/termos.

Conforme exposto nos referidos Termos e Condições de Uso, o descarregamento de títulos de acesso restrito requer uma licença válida de autorização devendo o utilizador aceder ao(s) documento(s) a partir de um endereço de IP da instituição detentora da supramencionada licença.

Ao utilizador é apenas permitido o descarregamento para uso pessoal, pelo que o emprego do(s) título(s) descarregado(s) para outro fim, designadamente comercial, carece de autorização do respetivo autor ou editor da obra.

Na medida em que todas as obras da UC Digitalis se encontram protegidas pelo Código do Direito de Autor e Direitos Conexos e demais legislação aplicável, toda a cópia, parcial ou total, deste documento, nos casos em que é legalmente admitida, deverá conter ou fazer-se acompanhar por este aviso.

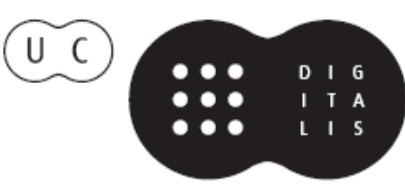


AVALIAÇÃO

$$
\text { FAMILIAR Vorstil Aorracicio }
$$

Q)

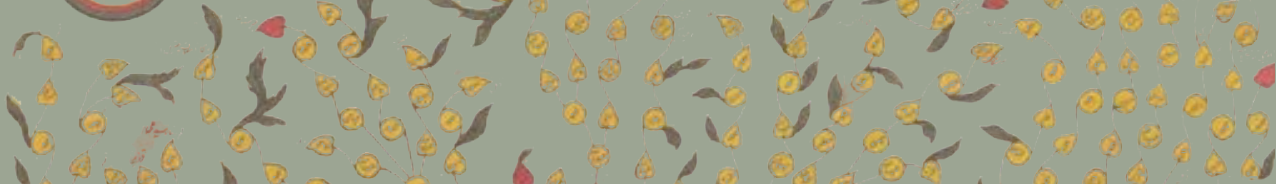

$-1000000$

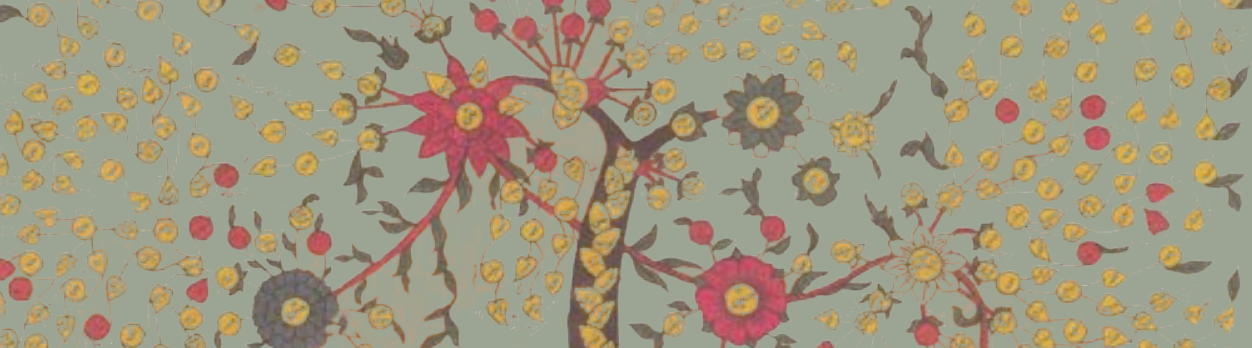

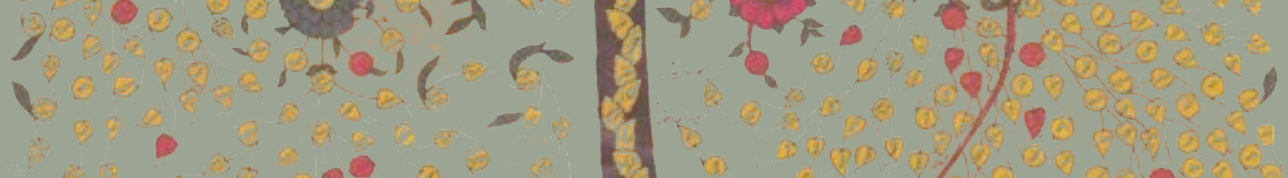

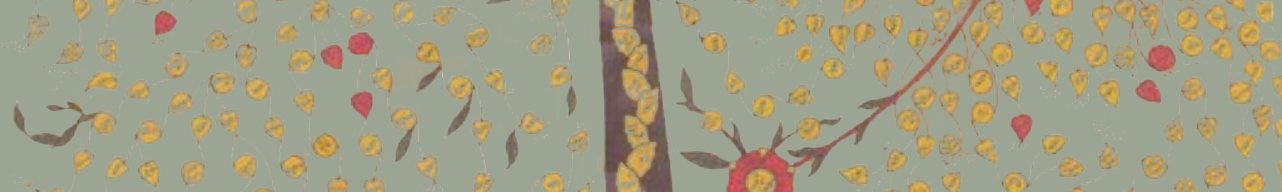

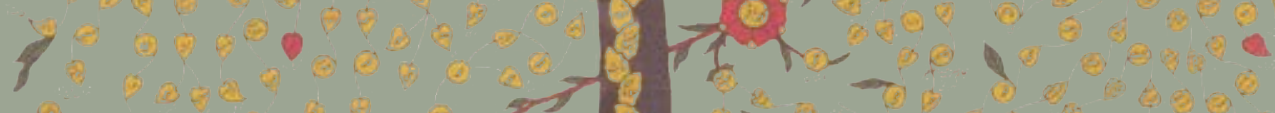

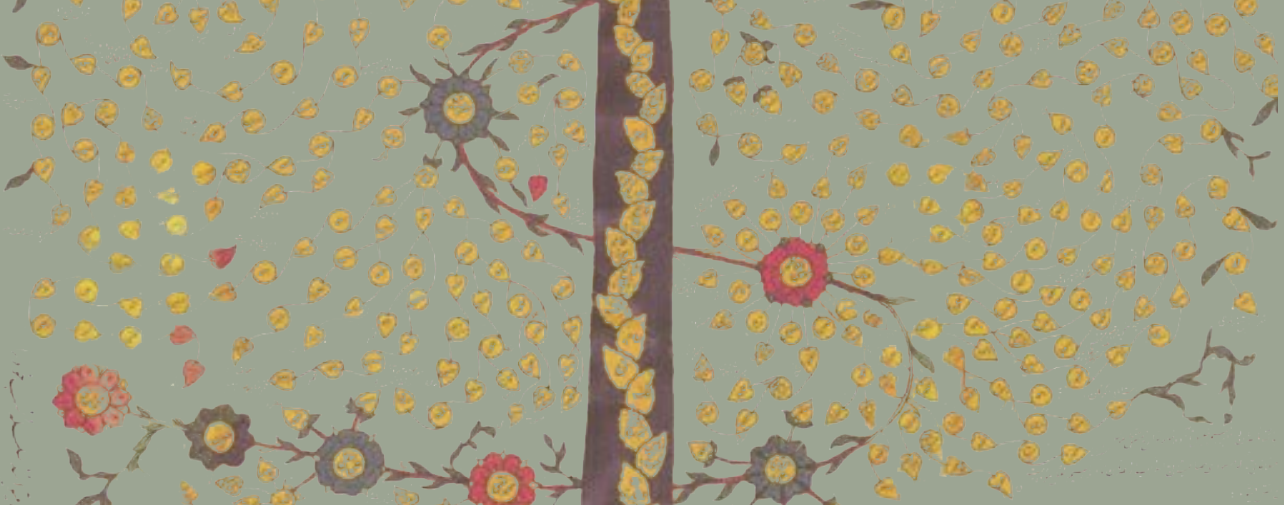

ANA PAULA RELVAS

SOFFA MAJOŔR Wh DE COIMBRA

COORDENAÇÃO 


\section{INVENTÁRIO DO LUTO PARA OS CUIDADORES DE MARWIT-MEUSER - FORMA REDUZIDA ( M M C G I - S F )}

Neide Areia

Sofia Major Ana Paula Relvas

"The anticipation of loss in physical illness can be as challenging and painful for families as the death of a family member."

(Rolland, 1990, p. 229)

\section{Resumo}

A doença oncológica traduz-se, frequentemente, num impacto significativo, não apenas para o doente mas, também, para todo o sistema familiar. Ao longo do ciclo de evolução da doença, o doente e sua família confrontam-se com perdas sucessivas que originam processos de luto antecipatório. Atendendo à relevância da compreensão do luto em familiares de doentes oncológicos, o presente estudo consiste na adaptação e validação do Marwit-Meuser Caregiver Grief Inventory - Short Form (MMCGI-SF; Marwit \& Meuser, 2005) a uma amostra portuguesa, constituída por 88 familiares de doentes com cancro em qualquer fase de evolução da doença. O MMCGI-SF é uma medida da experiência de luto antecipatório, constituída por 18 itens. Foi levado a cabo um estudo de análise fatorial exploratória tendo sido encontrados três fatores, não 
equivalentes à versão original. Relativamente aos estudos de consistência interna, obteve-se um bom coeficiente de alfa de Cronbach $(\alpha=.89)$ para a escala total. Foram analisadas as diferenças ao nível do resultado total no MMCGI-SF entre dois grupos de familiares de doentes em duas fases de tratamento da doença, ativo e paliativa, bem como entre grupos de familiares cuidadores principais e familiares não cuidadores. Os resultados apontaram para a inexistência de diferenças estatisticamente significativas. A versão portuguesa da MMCGI-SF representa, assim, uma medida de avaliação da experiência do luto antecipatório, enriquecedora do conjunto de instrumentos de avaliação da adaptação dos familiares/ cuidadores à doença crónica grave, disponíveis em Portugal, com características psicométricas satisfatórias que permitam a sua aplicação quer em contexto clínico, quer em investigação.

Palavras-chave: doença oncológica, familiares, luto antecipatório, avaliação, MMCGI-SF.

\section{Abstract}

Frequently, the oncologic disease has a profound impact not only on the patient, but also on the family systems. Through the illness' trajectory, patient and family face multiple losses that may originate experiences of anticipatory grief. Due to the importance of understand the grief experience of relatives of cancer patients, this study aims to adapt and validate the Marwit-Meuser Caregiver Grief Inventory - Short Form (MMCGI-SF; Marwit \& Meuser, 2005) to a Portuguese sample of 88 relatives of cancer patients, whatever the disease phase. The MMCGI-SF measures the anticipatory grief experience and has 18 items. An exploratory factor analysis was conducted in which three factors were found, not equivalent to the original version. The results of the internal consistency study, shows a good Cronbach alpha $(\alpha=.89)$ for the total scale. Differences in the MMCGI -SF total score for the groups of relatives of patients in active treatment phase and palliative care phase of the disease, as well as for the groups of 
primary family caregivers and non-caregivers were conducted. The results show that there were no statistically significant differences. The MMCGI-SF Portuguese version represents a measure of anticipatory grief, enriching the tools available that measures de relatives/caregivers adjustment to the chronic fatal disease, available in Portugal, with satisfactory psychometric characteristics that justify the use of it in clinical or research context.

Keywords: oncologic disease, relatives, anticipatory grief, assessment, MMCGI-SF.

\section{Instrumento}

O que é, o que avalia e a quem se aplica?

No Quadro 1 encontra-se a ficha técnica relativa ao Marwit-Meuser Caregiver Grief Inventory - Short Form (MMCGI-SF; Marwit \& Meuser, 2005).

Quadro 1.

Ficha técnica do MMCGI-SF

O Inventário do Luto para os Cuidadores de Marwit-Meuser (Forma Reduzida) é a versão portuguesa da Marwit-Meuser Caregiver Grief Inventory -

O que é? Short Form (MMCGI-SF), publicada em 2005, por Samuel Marwit e Thomas Meuser, em Washington, Estados Unidos da América. A versão original da MMCGI foi publicada em 2002

A MMCGI-SF consiste num questionário de auto-resposta, composto por 18 itens que avaliam a experiência de luto antecipatório nos familiares cuidadores de doentes oncológicos. Os 18 itens encontram-se repartidos por três dimensões: Sobrecarga e Sacrifício Pessoal, Sentimento de Tristeza e Saudade e Preocupação e Sentimento de Isolamento

\begin{tabular}{lcl}
\hline & Estrutura da MMCGI-SF (versão original) \\
\hline Subescala & Número Itens & Descrição \\
\hline $\begin{array}{l}\text { Sobrecarga } \\
\text { e Sacrifício } \\
\text { Pessoal }\end{array}$ & 6 & $\begin{array}{l}\text { Avalia as perdas (e.g., liberdade, independên- } \\
\text { cia) do indivíduo, experimentadas na sequên- } \\
\text { cia do acompanhamento do familiar doente }\end{array}$ \\
\hline $\begin{array}{l}\text { Sentimento } \\
\text { de Tristeza e }\end{array}$ & 6 & $\begin{array}{l}\text { Avalia a experiência de reações emocionais } \\
\text { (e.g., tristeza, sentimento de impotência) } \\
\text { decorrentes do acompanhamento do familiar } \\
\text { doente }\end{array}$ \\
\hline $\begin{array}{l}\text { Preocupaçãa } \\
\text { e Sentimento } \\
\text { de Isolamento }\end{array}$ & 6 & $\begin{array}{l}\text { Avalia o grau de incerteza relativamente à } \\
\text { evolução da doença e isolamento relativo à } \\
\text { rede social pessoal }\end{array}$ \\
\hline
\end{tabular}


A adaptação portuguesa da MMCGI-SF aplica-se a adultos ( $>18$ anos) familiares de doentes oncológicos em qualquer fase de evolução da doença. A quem se $\quad O$ inventário foi originalmente desenvolvido para cuidadores informais de aplica? doentes com Alzheimer (Marwit \& Meuser, 2002, 2005), tendo já sido validado junto de cuidadores de doentes com lesão cerebral (Marwit \& Kaye, 2006) e de cuidadores de doentes oncológicos (Marwit, Chibnall, Dougherty, Jenkins, \& Shawgo, 2008)

$\mathrm{O}$ acesso à versão portuguesa da MMCGI-SF pode ser efetuado através da página http://www.uc.pt/fpce/avaliacaofamiliar que contém todos os

Como ter acesso? instrumentos de avaliação apresentados neste livro. Os utilizadores deverão facultar os contactos pessoais e institucionais, bem como dados acerca do propósito da utilização do instrumento (e.g., investigação, prática clínica) e concordar com as condições de utilização e de partilha dos resultados com os autores da versão portuguesa

\section{Fundamentação e história}

A aproximação da morte, na sequência de uma doença crónica grave e progressiva afeta todo o sistema familiar (Tercero, 2002; Walsh \& McGoldrick, 2004). Envolve múltiplas perdas, não só de um membro do sistema, mas também de papéis e relações, de uma unidade familiar intacta, bem como, o desaparecimento de esperanças, sonhos e projetos para todos os membros da família. Assim, é expectável que a morte, factual ou eminente, seja considerada o desafio mais difícil que o sistema familiar tem de enfrentar (Walsh \& McGoldrick, 2004).

Neste sentido, a morte na família representa um fenómeno multidimensional (Gilbert, 1996), relacional e, ao mesmo tempo, individual, em que cada elemento da família experimenta o seu próprio luto (Wedemeyer, 1986). Importa entender o luto enquanto uma resposta intra e interpessoal, em que cada elemento do sistema sofre no contexto familiar (Gilbert, 1996) e a sua resposta influencia a do sistema e vice-versa (Brown, 1995; Kissane et al., 1996; Kissane \& Bloch, 2002; Moos, 1995; Walsh \& McGoldrick, 2004). Neste sentido, o luto familiar deve ser entendido com base no conhecimento dos fatores individuais e relacionais que operam simultaneamente e influem, naturalmente, na resposta familiar à perda. Em suma, o luto na família consiste numa experiência individual, de cada membro da família, num contexto relacional e social que é a própria família (Gilbert, 1996). O indivíduo, 
perante a morte do familiar, constrói a sua interpretação e perceção, que será influenciada pela cultura na qual está inserido, pela história familiar e funcionamento da família nuclear. Assim, a perceção de cada elemento da família influirá nos padrões de interação familiar que serão, necessariamente, alterados e constituirão a resposta familiar ao luto. Perante este fenómeno, a família ativa as suas estratégias de coping a fim de responder efetivamente à perda e recuperar o equilíbrio do sistema (Moos, 1995).

Perante o diagnóstico de uma doença ameaçadora da vida, e durante todo o curso da mesma, doente e familiares tendem a experimentar um processo de luto antecipatório (Guldin, Vedsted, Zachariae, Olesen, \& Jensen, 2012; Rolland, 1990), cuja expressão pode exacerbar-se aquando da progressão visível da doença ou aquando da comunicação de más notícias relacionadas com o prognóstico do doente (Lichtenthal, Prigerson, \& Kissane, 2010).

O luto antecipatório foi definido pela primeira vez por Lindemann (1944), reportando-se a uma convencional experiência de luto, aquando da existência de uma ameaça de morte (e.g., soldado na guerra). Assim, o indivíduo preocupado com o seu ajustamento à potencial morte de alguém que lhe seja significativo elabora todo um trabalho de luto; poderá manifestar sintomatologia depressiva, preocupação excessiva com a forma de morte do doente e antecipação da forma de ajustamento à sua ausência. Para este autor se, por um lado, experienciar um luto antecipatório pode servir de "amortecedor" face ao impacto da morte aquando da sua ocorrência, por outro, a elaboração completa deste tipo de luto pode criar distanciamento entre o enlutado e seu significativo (Lindemann, 1944). Sabemos atualmente que, ao descrever este fenómeno, Lindemann (1944) se referia à experiência de um luto prematuro (Zilberfein, 1999; Zisook, 2000 citado por Grassi, 2007).

Mais recentemente, Grassi (2007) define luto antecipatório como uma forma de a família, ao antecipar a perda do seu membro, se preparar para trabalhar o trauma da morte e o seu ajustamento após a ocorrência da mesma. Por outras palavras, o luto antecipatório consiste na experiência de um processo de luto antes da ocorrência da morte (Reynolds \& Botha, 2006). 
Apenas recentemente se tem dado relevância à experiência de luto antes da ocorrência da morte. Nesta linha, Marwit e Meuser (2002) desenvolveram o Marwit-Meuser Caregiver Grief Inventory (MMCGI) para avaliar a experiência de luto nos cuidadores de doentes com Alzheimer. Dos estudos de análise fatorial, resultou um instrumento composto por 50 itens, divididos em três dimensões: Sobrecarga e Sacrifício Pessoal (18 itens), Sentimento de Tristeza e Saudade (15 itens) e Preocupação e Sentimento de Isolamento (17 itens). O MMCGI foi desenvolvido a partir de uma amostra de 166 cuidadores de doentes com Alzheimer, sendo que 135 eram mulheres e apenas 31 eram homens. O instrumento apresentou um coeficiente de alfa de Cronbach de .96 para a escala total. Relativamente às subescalas, todas apresentaram um alfa de Cronbach de .90 ou superior (Sobrecarga e Sacrifício Pessoal $=.93$; Sentimento de Tristeza e Saudade $=.90 ;$ Preocupação e Sentimento de Isolamento $=.90$ ) .

O MMCGI foi, ainda, aplicado a 28 cuidadores de doentes com lesão cerebral, tendo um índice de fiabilidade superior a .90 ao nível da escala total e subescalas (Marwit \& Kaye, 2006). Finalmente, também com a finalidade de avaliar as características psicométricas do MMCGI aquando da sua utilização com cuidadores de doentes oncológicos, foi desenvolvido um estudo junto de uma amostra de 75 sujeitos cuidadores de doentes neoplásicos. Verificou-se, também para esta amostra, que os itens do MMCGI apresentam um elevado nível de consistência interna ao considerar a totalidade da escala $(\alpha=.96)$ e respetivas subescalas (Sobrecarga e Sacrifício Pessoal $=.95$; Sentimento de Tristeza e Saudade $=.94$; e Preocupação e Sentimento de Isolamento $=.90$ ). Importa destacar que os cuidadores de doentes oncológicos revelaram índices de luto inferiores aos cuidadores de doentes com lesão cerebral e cuidadores de doentes com Alzheimer (Marwit et al., 2008).

Com a finalidade de reduzir o tempo de preenchimento do instrumento e, no sentido de facilitar a sua integração em protocolos mais longos, foi desenvolvida uma versão reduzida do MMCGI. O Marwit Meuser Caregiver Grief Inventory - Short Form (MMCGI-SF; Marwit \& Meuser, 2005) é uma medida da experiência de luto antecipatório e é constituído por 18 itens. Cada item é cotado de acordo com uma escala do tipo Likert com cinco níveis de resposta: 1 = "Discordo fortemente"; 2 = "Discordo"; 
3 = "Concordo moderadamente"; 4 = "Concordo"; 5 = "Concordo fortemente". De igual modo, o MMCGI-SF contempla as dimensões Sobrecarga e Sacrifício Pessoal (6 itens) (e.g., "Tive que desistir de ..."), Sentimento de Tristeza e Saudade (6 itens) (e.g., "Tenho este desagradável sentimento..."), Preocupação e Sentimento de Isolamento (6 itens) (e.g., "Não tenho ninguém com ..."). Com a finalidade de testar as características psicométricas do instrumento e verificar a sua equivalência com a versão original, foi realizado um estudo junto de uma amostra de 292 cuidadores de doentes com diagnóstico de demência de Alzheimer. Nesta investigação, obteve-se um valor de coeficiente de alfa de Cronbach de .90 para a escala total, .83 para a subescala Sobrecarga e Sacrifício Pessoal, .80 para a subescala Sentimento de Tristeza e Saudade e .80 para a subescala Preocupação e Sentimento de Isolamento (Marwit \& Meuser, 2005).

Importa destacar que no referido estudo, e até à data, não foram realizados estudos de análise fatorial com o MMCGI-SF de modo a verificar eventuais discrepâncias na sua estrutura fatorial comparativamente à versão original (Marwit \& Meuser, 2005).

\section{Estudos em Portugal}

Como foi desenvolvido/adaptado e validado?

\section{Estudos de tradução e adaptação}

Após o pedido formal de autorização junto de um dos autores do MMCGI-SF (Thomas Meuser, University of Missouri - St. Louis) para tradução e adaptação da escala, deu-se início, em setembro de 2012, ao processo de tradução-retroversão (Gjersing, Caplehorn, \& Clausen, 2010). Num primeiro momento, foram desenvolvidas duas traduções, de modo independente, por dois investigadores fluentes em português e inglês. De seguida, tendo como ponto de partida as traduções iniciais, um terceiro investigador, com competências linguísticas semelhantes, procedeu a uma tradução conciliadora das anteriores. 
Realizou-se um estudo preliminar do instrumento, de modo a avaliar a sua adequação semântica. A versão final preliminar foi então administrada a 12 sujeitos, familiares de doentes oncológicos, tendo sido pedido que registassem eventuais erros, desadequações, ambiguidades na formulação dos itens ou outras dificuldades sentidas ao responder ao MMCGI-SF. Os itens 4 (primeira tradução: "Tenho este desagradável sentimento de vazio por saber que o meu familiar 'partiu'.") e 11 (primeira tradução: "Custa-me deitá-lo(a) na cama e perceber que ele(a) 'partiu'.") foram contestados pelos familiares. Alguns respondentes alegaram que, atendendo à condição clínica do doente, estes itens não seriam aplicáveis uma vez que, ainda numa fase muito incipiente da evolução da doença, em que existe expetativa de cura, lhes era difícil imaginar o doente com prognóstico limitado. Por outro lado, um doente oncológico, na maioria dos casos, preserva a sua função cognitiva, pelo que este "partir", ainda que simbolicamente, não seria adequado. Estas questões foram discutidas com os autores da escala que anuíram a proposta de alteração da tradução (item 4: "Tenho este desagradável sentimento de vazio por saber que o meu familiar vai 'partir'."; item 11: "Custa-me deitá-lo(a) na cama e perceber que ele(a) vai 'partir'."). Por fim, efetuados os acertos finais na versão portuguesa do MMCGI-SF, foi realizada uma retroversão do instrumento para inglês, por um professor de inglês. A retroversão da escala foi enviada aos autores originais que, após análise, validaram a tradução portuguesa e a utilização e adaptação da escala para uma amostra de familiares - cuidadores e não cuidadores - de doentes oncológicos, em qualquer fase de evolução da doença.

Obtida a versão traduzida da MMCGI-SF - Inventário de Luto para Cuidadores (versão breve) - iniciaram-se os estudos de validação do instrumento.

Dos 47 pedidos de autorização formalizados para os conselhos de administração e comissões de ética das instituições médicas, foram obtidas quatro autorizações para a recolha da amostra (Hospital de Braga, Hospital de Beja, Hospital de Cantanhede, Instituto Português de Oncologia de Coimbra) (cf. capítulo 5).

No que diz respeito à constituição da amostra, consideraram-se os seguintes critérios de inclusão/ exclusão: a) ser familiar de um doente 
oncológico em qualquer fase de evolução da doença (e.g., cônjuge, filho/a, irmão/ã, pai, mãe, neto/a, primo/a), b) ter mais de 18 anos, c) saber ler e escrever, e d) não apresentar psicopatologia não compensada.

A recolha da amostra decorreu de março de 2013 a março de 2015. Para efeitos de administração do protocolo, os familiares foram questionados pelo médico que acompanhava o doente ou por outro técnico (e.g., psicólogo ou técnico de serviço social), acerca do seu interesse em participar no estudo. Caso estivessem de acordo em participar no mesmo, os familiares eram encaminhados para uma sala reservada para o efeito, onde se encontrava a investigadora responsável. Aqui foram fornecidas informações mais detalhadas sobre a investigação, esclarecidas as questões dos familiares e garantida a confidencialidade e anonimato dos dados fornecidos e procedeu-se à assinatura do documento de consentimento informado. Após o preenchimento do consentimento informado, a investigadora administrava o protocolo de avaliação em formato de entrevista e esclarecia devidamente todas as questões que surgissem durante a administração do protocolo.

Numa primeira fase, foi realizado um estudo de natureza exploratória junto de uma amostra de $\mathbf{4 1}$ familiares de doentes oncológicos em diversas fases de evolução da doença. Neste âmbito, foi levado a cabo um estudo de análise fatorial exploratória, tendo sido encontrados três fatores, coincidindo a maioria dos itens com os da versão original da MMCGI-SF. Relativamente aos estudos de precisão/consistência interna, obteve-se um bom coeficiente de alfa de Cronbach $(\alpha=.89)$ para a escala total (Correia, 2014).

Para o presente estudo, a amostra é composta por 88 sujeitos, maioritariamente do sexo feminino (69.3\%), com idades compreendidas entre os 18 e os 82 anos, com uma média de idades de 46.72 ( $D P=15.96$ ) (cf. Quadro 2). A escolaridade dos participantes é diversificada sendo o $3 .^{\circ}$ ciclo do ensino básico a mais comum (26.1\%). Quanto ao estado civil, a maioria dos sujeitos são casados (68.2\%). No que diz respeito à região de residência, a maioria dos sujeitos reside na região centro do país (60.2\%) e, em particular, no distrito de Coimbra (34.1\%).

No que diz respeito ao papel desempenhado relativo à prestação de cuidados, mais de metade dos sujeitos considera ser o cuidador principal do doente (62.5\%). A maioria dos respondentes referem prestar mais de 
11 horas de cuidados (33.0\%). Os sujeitos são, maioritariamente, filhos (38.6\%) ou cônjuges (31.8\%) do doente.

Quadro 2.

Caracterização da amostra (familiares)

\begin{tabular}{|c|c|c|c|}
\hline & & Frequência $(n)$ & Percentagem (\%) \\
\hline \multirow{2}{*}{ Sexo } & Masculino & 27 & 30.7 \\
\hline & Feminino & 61 & 69.3 \\
\hline \multirow{6}{*}{ Faixa etária } & $18-29$ & 12 & 13.6 \\
\hline & $30-39$ & 22 & 25.0 \\
\hline & $40-49$ & 18 & 20.4 \\
\hline & $50-59$ & 14 & 15.9 \\
\hline & $60-69$ & 16 & 18.2 \\
\hline & $70-82$ & 6 & 6.8 \\
\hline \multirow{6}{*}{ Escolaridade } & Sem escolaridade & 1 & 1.1 \\
\hline & $1^{\circ}$ Ciclo do ensino básico & 19 & 21.6 \\
\hline & $2^{\circ}$ Ciclo do ensino básico & 7 & 8.0 \\
\hline & $3^{\circ}$ Ciclo do ensino básico & 23 & 26.1 \\
\hline & Ensino secundário & 19 & 21.6 \\
\hline & Ensino superior & 19 & 21.6 \\
\hline \multirow{5}{*}{ Estado civil } & Solteiro & 19 & 21.6 \\
\hline & Casado/União de facto & 60 & 68.2 \\
\hline & Recasado & 1 & 1.1 \\
\hline & Divorciado & 6 & 6.8 \\
\hline & Viúvo & 2 & 2.3 \\
\hline \multirow{3}{*}{ Região } & Norte & 20 & 22.7 \\
\hline & Centro & 53 & 60.2 \\
\hline & Sul & 15 & 17.0 \\
\hline \multirow{11}{*}{ Distrito } & Aveiro & 8 & 9.1 \\
\hline & Beja & 14 & 15.9 \\
\hline & Braga & 20 & 22.7 \\
\hline & Coimbra & 30 & 34.1 \\
\hline & Castelo Branco & 2 & 2.3 \\
\hline & Guarda & 1 & 1.1 \\
\hline & Leiria & 6 & 6.8 \\
\hline & Lisboa & 1 & 1.1 \\
\hline & Portalegre & 1 & 1.1 \\
\hline & Santarém & 2 & 2.3 \\
\hline & Viseu & 3 & 3.4 \\
\hline
\end{tabular}




\begin{tabular}{llcc}
\hline Cuidador & Principal & 55 & 62.5 \\
& Secundário/Não cuidador & 33 & 37.5 \\
& & & \\
Horas & a 2 horas & 9 & 10.2 \\
de cuidados & 3 a 5 horas & 16 & 18.2 \\
(diárias) & 6 a 8 horas & 9 & 10.2 \\
& 9 a 11 horas & 6 & 6.8 \\
& Mais de 11 horas & 29 & 33.0 \\
& Não presta cuidados & 19 & 21.6 \\
Relação com & Filho/a & & \\
o doente & Cônjuge & 34 & 38.6 \\
& Outra & 28 & 31.8 \\
& & 26 & 29.5 \\
\hline
\end{tabular}

Relativamente ao doente, a média de idades é 62.88 anos $(D P=15.59)$, com idades compreendidas entre os 21 e os 86 anos. O diagnóstico oncológico é muito diversificado, sendo o cancro de mama (25.0\%) e o cancro do cérebro (11.4\%) os mais comuns. Mais de metade dos doentes recebeu o diagnóstico há menos de um ano (62.5\%) e 40 doentes apresentam doença em fase terminal da sua evolução (cf. Quadro 3).

Quadro 3.

Caracterização da amostra (doente e doença)

\begin{tabular}{|c|c|c|c|}
\hline & & Frequência $(n)$ & Percentagem (\%) \\
\hline \multirow{7}{*}{ Faixa etária } & $21-29$ & 4 & 4.5 \\
\hline & $30-39$ & 3 & 3.4 \\
\hline & $40-49$ & 9 & 10.2 \\
\hline & $50-59$ & 14 & 15.9 \\
\hline & $60-69$ & 26 & 29.5 \\
\hline & $70-79$ & 19 & 21.6 \\
\hline & $80-86$ & 13 & 14.8 \\
\hline \multirow{2}{*}{$\begin{array}{l}\text { Contexto de presta- } \\
\text { ção de cuidados }\end{array}$} & Internamento & 21 & 23.9 \\
\hline & Ambulatório & 67 & 76.1 \\
\hline \multirow{5}{*}{$\begin{array}{l}\text { Tempo desde o } \\
\text { diagnóstico }\end{array}$} & 0 a 1 anos & 55 & 62.5 \\
\hline & 2 a 4 anos & 24 & 27.3 \\
\hline & 5 a 7 anos & 5 & 5.7 \\
\hline & 8 a 10 anos & 3 & 3.4 \\
\hline & Mais de 11 anos & 1 & 1.1 \\
\hline
\end{tabular}




\begin{tabular}{llcc}
\hline \multirow{3}{*}{ Fase da Doença } & Crise & 11 & 12.5 \\
& Crónica & 29 & 33.0 \\
& Recidiva & 4 & 4.5 \\
& Terminal & 40 & 45.5 \\
& Remissão & 4 & 4.5 \\
\multirow{6}{*}{ Diagnóstico } & & & \\
& Mama & 22 & 25.0 \\
& Cérebro & 10 & 11.4 \\
& Útero & 7 & 8.0 \\
& Outros & 49 & 55.6 \\
\hline
\end{tabular}

Estudos de análise de itens: Estatísticas descritivas

No Quadro 4 encontram-se os resultados referentes às estatísticas descritivas para os 18 itens do MMCGI-SF.

De acordo com o Quadro 4, o item 12 ("Sinto-me muito triste...") é o que apresenta a média mais elevada $(M=4.35, D P=1.03)$. Por sua vez, o item com a média mais baixa $(M=1.84, D P=1.08)$ é o item 9 ("Conseguiria lidar melhor ..."). Relativamente à amplitude, todos os itens atendem a toda a amplitude da escala de cotação (1 - "Discordo fortemente" a 5 - "Concordo fortemente").

Quadro 4.

Estatísticas descritivas: Itens do MMCGI-SF

\begin{tabular}{lccccc}
\hline Item & $M$ & $D P$ & Mín-Máx & Assimetria & Curtose \\
\hline 1 & 2.81 & 1.52 & $1-5$ & 0.19 & -1.37 \\
2 & 2.42 & 1.52 & $1-5$ & 0.62 & -1.12 \\
3 & 2.11 & 1.29 & $1-5$ & 0.87 & -0.43 \\
4 & 3.8 & 1.46 & $1-5$ & -0.82 & -0.83 \\
5 & 3.61 & 1.38 & $1-5$ & -0.60 & -0.98 \\
6 & 2.07 & 1.30 & $1-5$ & 1.02 & -0.15 \\
7 & 3.72 & 1.40 & $1-5$ & 0.80 & -0.86 \\
8 & 3.72 & 1.40 & $1-5$ & -0.72 & -0.86 \\
9 & 1.84 & 1.08 & $1-5$ & 1.22 & 0.82 \\
10 & 3.81 & 1.32 & $1-5$ & -1.01 & -0.08 \\
11 & 3.49 & 1.69 & $1-5$ & -0.57 & -1.42 \\
12 & 4.35 & 1.03 & $1-5$ & -1.73 & 2.51 \\
13 & 3.5 & 1.51 & $1-5$ & -0.49 & -1.25 \\
\hline
\end{tabular}




\begin{tabular}{llllll}
\hline 14 & 2.20 & 1.34 & $1-5$ & 0.79 & -0.59 \\
15 & 3.59 & 1.54 & $1-5$ & -0.63 & -1.17 \\
16 & 2.49 & 1.55 & $1-5$ & 0.51 & -1.27 \\
17 & 2.62 & 1.48 & $1-5$ & 0.35 & -1.31 \\
18 & 2.33 & 1.46 & $1-5$ & 0.68 & -1.01 \\
\hline
\end{tabular}

Analisando os valores referentes à assimetria observa-se que os itens $6,9,10$ e 12 são aqueles que se afastam mais de zero. Observando os valores da curtose, verifica-se que o maior afastamento na distribuição dos resultados se verifica nos itens 1,11 e 12 .

\section{Estudos de precisão: Consistência interna dos itens do MMCGI-SF}

Para o presente estudo foi realizada uma análise de consistência interna para os 18 itens do MMCGI-SF para averiguar o grau de confiabilidade do instrumento (cf. Quadro 5).

Quadro 5.

Estudo de consistência interna dos itens do MMCGI-SF

\begin{tabular}{lcc}
\hline Itens & Correlação item-total corrigida & Alfa com eliminação do item \\
\hline 1 & .61 & .88 \\
2 & .71 & .88 \\
3 & .48 & .89 \\
4 & .60 & .88 \\
5 & .69 & .88 \\
6 & .56 & .88 \\
7 & .32 & .89 \\
8 & .52 & .88 \\
9 & .31 & .89 \\
10 & .47 & .89 \\
11 & .62 & .88 \\
12 & .55 & .88 \\
13 & .52 & .88 \\
14 & .24 & .89 \\
15 & .36 & .89 \\
16 & .68 & .88 \\
17 & .57 & .88 \\
18 & .60 & .88 \\
\hline
\end{tabular}


No que respeita à escala total, obtivemos um coeficiente de alfa de Cronbach de .89, o que nos remete para um bom índice de consistência interna (Pestana \& Gageiro, 2008). De forma a verificar se a eliminação de algum item levaria a um aumento do valor do alfa de Cronbach, foi analisado o valor das correlações item-total corrigidas e do valor do alfa de Cronbach caso o item fosse eliminado. Apenas o item 14 apresentou uma correlação inferior a $.30(r=.24)$. Porém, a sua exclusão não traduziria um aumento importante do coeficiente de alfa de Cronbach, pelo que se optou por manter o item.

\section{Estudos de validade interna: Análise fatorial exploratória (AFE)}

Num primeiro momento, para se verificar a adequação dos pressupostos para a realização da AFE, verificou-se a fatoriabilidade da amostra, através da estimação do teste de Kaiser-Meyer-Olkin (KMO) e do teste de esfericidade de Bartlett, com o objetivo de aferir a qualidade das correlações entre as variáveis. O resultado obtido no teste de KMO (.856) revelou uma boa adequação da amostra para uma análise em componentes principais (Pestana \& Gageiro, 2008). No que diz respeito ao teste de esfericidade de Bartlett, obteve-se um valor estatisticamente significativo $\left(\chi^{2}=857.484 ; g l=153 ; p<.001\right)$. Deste modo, e uma vez que a matriz de correlações não é uma matriz de identidade, as variáveis em estudo são correlacionáveis.

Verificados os pressupostos, efetuou-se uma análise fatorial de componentes principais, onde foi sugerida a extração de 3 componentes que explicam $60.51 \%$ da variância total. Do mesmo modo, da análise do scree-plot, verifica-se um ponto de inflexão no quarto componente, remetendo para a retenção de três fatores.

De seguida, de acordo com a estrutura original do instrumento, procedeu-se à rotação varimax forçada a três fatores. Assim, de acordo com o Quadro 6, o primeiro fator é composto por sete itens - itens 1, 2, 3, 6, 16, 17 e 18 - que explicam 25.92\% da variância total, com valores de saturação que oscilam entre .509 e .914. 
A análise dos itens que compõem este fator indica que cinco itens pertencem à subescala Sobrecarga e Sacrifício Pessoal da versão original do MMCGI-SF. Não se obteve correspondência para os itens 3 e 6, ambos pertencentes ao fator 3 na versão original. Importa salientar que o item 3 apresenta um valor de saturação de .585 no fator 1 e .378 no fator 3. Atendendo ao conteúdo teórico do item 3, optou-se por integrá-lo no fator 3: Preocupação e Sentimento de Isolamento, ficando assim o primeiro fator composto por seis itens.

Quadro 6.

Matriz rodada a três fatores dos itens do MMCGI-SF, variância explicada e comunalidades (rotação Varimax)

\begin{tabular}{|c|c|c|c|c|}
\hline Itens & & mpone & & $b^{2}$ \\
\hline & 1 & 2 & 3 & \\
\hline 16. Independência foi o que... & .914 & & & .835 \\
\hline 1. Tive que desistir de... & .897 & & & .804 \\
\hline 2. Sinto que estou... & .859 & & & .738 \\
\hline 18. Estou preso neste mundo... & .832 & & & .692 \\
\hline 17. Desejava ter... & .721 & & & .520 \\
\hline 3. Não tenho ninguém com ... & .585 & & .378 & .485 \\
\hline 6. A doença é como... & .509 & & & .259 \\
\hline 5. Passo muito tempo preocupado... & & .845 & & .714 \\
\hline 11. Custa-me deitá-lo(a)... & & .808 & & .653 \\
\hline 12. Sinto-me muito triste... & & .803 & & .645 \\
\hline 4. Tenho este desagradável sentimento... & & .803 & & .645 \\
\hline 13. Fico acordado(a) a maioria... & & .779 & & .607 \\
\hline 15. Já perdi outras pessoas... & & .592 & & .352 \\
\hline 8. Sinto saudades... & .368 & .506 & & .391 \\
\hline 10. Ninguém sabe até quando... & .389 & .390 & & .303 \\
\hline 14. As pessoas que me... & & & .838 & .702 \\
\hline 7. Os meus amigos... & & & .793 & .674 \\
\hline 9. Conseguiria lidar melhor... & & & .529 & .363 \\
\hline \% variância explicada & 25.92 & 23.94 & 10.65 & \\
\hline
\end{tabular}

O segundo fator é composto por oito itens - itens 4, 5, 8, 10, 11, 12, 13 e 15 - que explicam 23.94\% da variância total, com valores de saturação que oscilam entre .390 e .845. A análise dos itens que compõe este fator indica que cinco itens pertencem à subescala Sentimento de Tristeza e Saudade da versão original. Dos três itens para os quais não se obteve correspondência, dois pertencem ao fator 3 da versão original (itens 5 e 13) e o item 10 ao fator 1 (cf. Quadro 6). 
Finalmente, o terceiro fator é composto por três itens - itens 7, 9 e 14 que explicam $10.65 \%$ da variância total, com valores de saturação que oscilam entre .529 e .838 (cf. Quadro 6). A análise dos itens que compõe este fator indica que dois itens pertencem à subescala Preocupação e Sentimento de Isolamento da versão original (itens 7 e 14). O item 9 pertencia ao fator 2 na versão original. Com a fixação do item 3 neste fator, o terceiro fator passou a integrar quatro itens.

Assim, a solução fatorial resultante apresenta uma correspondência de 13 itens com a versão original, no total de 18, distribuídos pelos três fatores. Importa salientar que não existem, até então, estudos de análise fatorial com a versão breve do MMCGI (Marwit \& Meuser, 2005), uma vez que esta versão foi desenvolvida com base nos itens com saturação mais elevada da versão de 50 itens de Marwit e Meuser (2002).

Estudos de validade interna: Análise fatorial confirmatória (AFC)

No sentido de verificar a adequação do modelo da versão original foram realizados estudos de AFC para os 18 itens do MMCGI-SF tendo por base a estrutura fatorial da versão original (Marwit \& Meuser, 2005). Para tal, recorreu-se ao software AMOS 22 (Analysis of Moment Structures) e utilizou-se o método de estimação de máxima verosimilhança (Maximum Likelihood). O ajustamento do modelo foi verificado tendo em consideração os seguintes índices: Qui-quadrado/ graus de liberdade ( $\left.\chi^{2} / \mathrm{df}\right)$, CFI (Comparative Fit Index), GFI (Goodnessof-fit Index) e RMSEA (Root Mean Square Error of Aproximation) (Marôco, 2010). Os valores considerados para o ajustamento do modelo foram: $\chi^{2} / d f$ inferior a 5, CFI e GFI superiores a .90 e RMSEA inferior a .10 (Marôco, 2010).

A Figura 1 representa o modelo proposto por Marwit e Meuser (2005), com correlações entre os três fatores a oscilar entre .41 (Sobrecarga_Sacrifício e Tristeza_Saudade) e .96 (Tristeza_Saudade e Preocupação_Isolamento). As saturações estandardizadas para os 
itens dos três fatores considerados oscilam entre .181 e .938. O modelo original não se demonstrou ajustado para os dados recolhidos, uma vez que os valores obtidos para os índices de ajustamento não alcançaram os valores de referência: $\chi^{2} / d f=1.699, C F I=.881, G F I$ $=.778$ e RMSEA $=.090$ (IC = .069-.109). A análise dos índices de modificação também não apontou para uma melhoria do modelo.

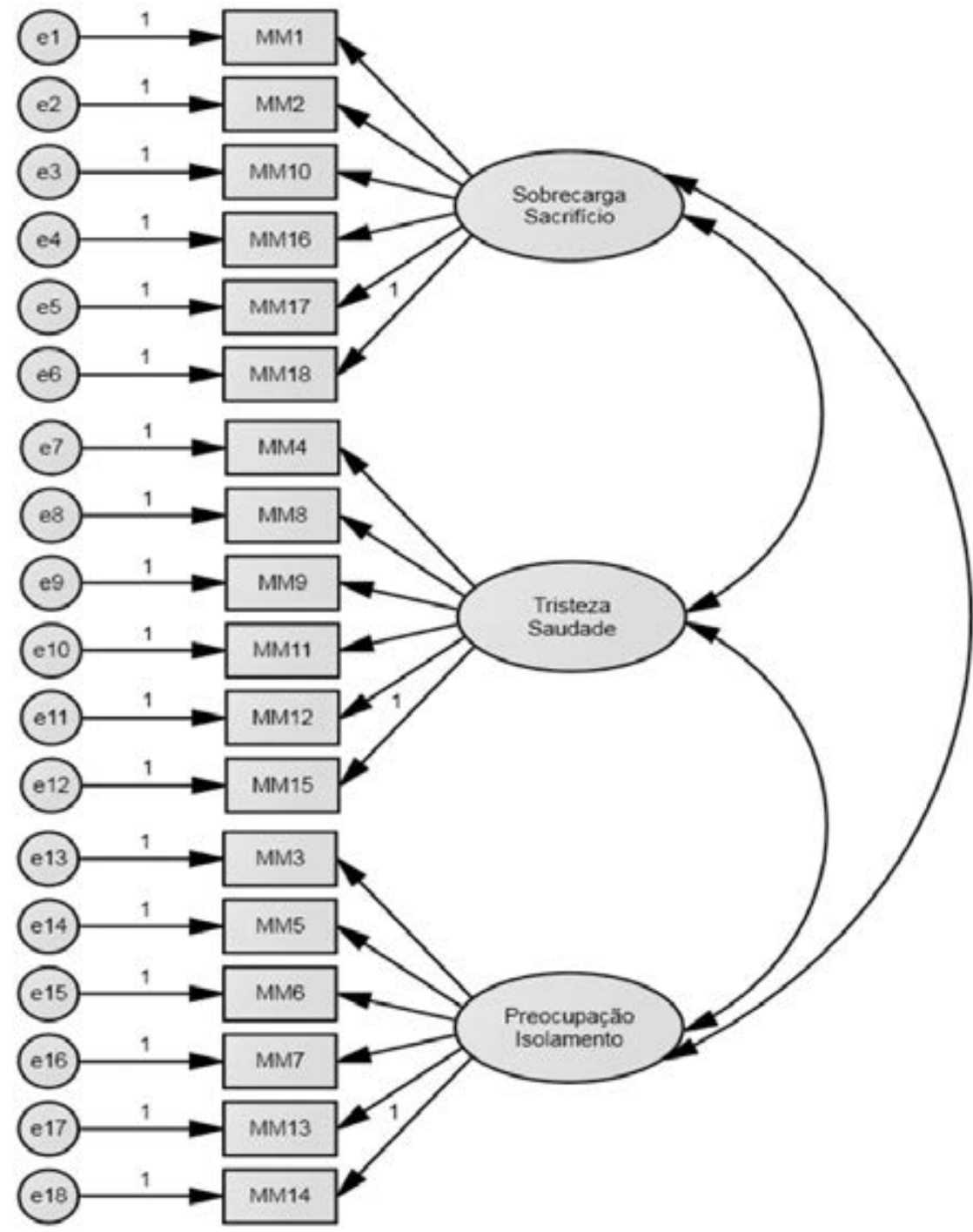

Figura 1. Modelo MMCGI-SF versão original (Marwit \& Meuser, 2005). 
Por conseguinte, e dado o tamanho reduzido da amostra $(n=88)$, optou-se por considerar nos vários estudos realizados apenas o resultado total da escala - Nível de Luto Total.

Estudos de validade com grupos conhecidos: Grupos de familiares de doentes em fases de tratamento ativo e paliativa da doença e grupos de familiares cuidadores principais e não cuidadores

Procurou-se analisar a existência de diferenças ao nível do Luto Total (resultado total do MMCGI-SF) entre o grupo de familiares de doentes em fase de tratamento ativo da doença (fase de crise, crónica, recidiva e remissão) e fase de tratamento paliativo (fase terminal), assim como entre o grupo de familiares cuidadores principais e familiares não cuidadores. Para o efeito, recorreu-se ao teste $t$ de Student para amostras independentes.

Não foram encontradas diferenças estatisticamente significativas entre os familiares de doentes em fase de tratamento ativo e paliativo ao nível do Luto Total, $t(85)=-3.606, p=.748$. Do mesmo modo, não se verificaram diferenças entre os familiares cuidadores principais e os familiares não cuidadores ao nível do Luto Total, $t(85)=3.089$, $p=.711$.

\section{Aplicação}

\section{Como aplicar, cotar e interpretar?}

O material necessário para a aplicação da MMCGI-SF é apenas a versão em papel do questionário e uma caneta. A aplicação da MMCGI-SF requer que o sujeito cote cada um dos 18 itens relativamente ao seu grau de concordância com os itens. Os itens são cotados numa escala de tipo Likert, de 1 ("Discordo fortemente") a 5 ("Concordo fortemente").

Este estudo exploratório permitiu calcular os primeiros valores de referência para a MMCGI-SF em Portugal. Assim, no Quadro 7 apresentam-se as médias e desvios-padrão para o resultado total da escala, 
o que corresponde ao nível de Luto Total. Apresentam-se, ainda, os valores de referência para o grupo de familiares de doentes em fase de tratamento ativo e paliativo.

Quadro 7.

Valores de referência da MMCGI-SF ao nível do Luto Total

\begin{tabular}{|c|c|c|c|c|c|c|}
\hline \multirow[t]{2}{*}{ Resultados MMCGI-SF } & \multicolumn{2}{|c|}{$\begin{array}{l}\text { Tratamento ativo } \\
\qquad(n=48)\end{array}$} & \multicolumn{2}{|c|}{$\begin{array}{l}\text { Tratamento paliativo } \\
\qquad(n=40)\end{array}$} & \multicolumn{2}{|c|}{$\begin{array}{l}\text { Amostra Total } \\
\qquad(N=88)\end{array}$} \\
\hline & $M$ & $D P$ & $M$ & $D P$ & $M$ & $D P$ \\
\hline Nível de Luto Total & 48.02 & 14.31 & 58.95 & 13.82 & 53.05 & 15.04 \\
\hline
\end{tabular}

Relativamente à interpretação dos resultados, importa referir que resultados elevados no resultado total indicam possíveis dificuldades na adaptação à doença e, por conseguinte, eventual necessidade de suporte psicoterapêutico formal. Por seu turno, resultados baixos no resultado total indicam uma possível adaptação funcional à doença. Neste último caso, deve despistar-se se o sujeito se encontra, ou não, em negação relativamente à doença e eventual eminência da morte.

\section{Vantagens, limitações e estudos futuros}

O estudo do luto antecipatório é, ainda, incipiente (Kissane \& Bloch, 2002). As medidas que visam avaliar a experiência de luto antecipatório nos familiares de doentes crónicos com doença ameaçadora de vida são, ainda, muito escassas (Marwit \& Kaye, 2006; Marwit \& Meuser, 2002; Marwit et al., 2008).

O MMCGI-SF visa medir a experiência de luto antecipatório de familiares de doentes oncológicos e revela ser um instrumento com propriedades psicométricas razoáveis. Ao nível clínico, o MMCGI-SF permite sinalizar os familiares - cuidadores, ou não - que apresentem níveis de luto preocupantes e, eventualmente, associados ao desenvolvimento de luto complicado e patológico no pós-morte e, por conseguinte, encaminhar para o tipo de suporte mais adequado (e.g., psiquiatria, psicologia). 
Ao nível da investigação, fica disponível para a população portuguesa uma ferramenta de estudo da experiência de luto antecipatório, ao permitir que seja dado incremento à investigação numa área, comumente, menos valorizada.

As limitações deste estudo de adaptação do MMCGI-SF prendem-se com as características da amostra (amostra não probabilística de conveniência e não estratificada e de dimensão relativamente reduzida) e a não replicação da estrutura fatorial original do instrumento.

Deste modo, seria interessante aprofundar o estudo do funcionamento psicométrico do MMCGI-SF, realizando, após a devida ampliação da amostra, estudos de análise fatorial confirmatória de modo a averiguar a correspondência dos itens à estrutura original proposta pelos autores do instrumento (Marwit \& Meuser, 2002).

Parece-nos ainda que os estudos de adaptação e validação do MMCGI-SF beneficiariam de análises independentes com populações clínicas diferentes, de modo a avaliar a capacidade discriminante do questionário e desenvolver valores normativos para as demais populações. Nesta linha, é crucial identificar eventuais diferenças na experiência de luto antecipatório em diferentes fases da evolução da doença (e.g., crise, crónica, terminal), diferentes diagnósticos oncológicos (e.g., mama, cérebro, pulmão, leucemia) e diferentes diagnósticos de doença crónica ameaçadora da vida (e.g., insuficiências de órgão, doença de Alzheimer, SIDA). Importa, ainda, desenvolver estudos que analisem a influência da experiência do luto antecipatório na adaptação individual (e.g., sintomatologia psiquiátrica, necessidades) e familiar (e.g., funcionamento e coping familiar) à doença e proximidade da morte.

\section{Bibliografia}

Brown, F. (1995). O impacto da morte e da doença grave sobre o ciclo de vida familiar. In B. Carter, \& M. McGoldrick (Eds.). As mudanças no ciclo de vida familiar. Uma estrutura para a terapia familiar (2 ${ }^{\mathrm{a}}$ ed., pp. 393-413). Porto Alegre: Artes Médicas.

Correia, M. (2014). Luto antecipatório na doença oncológica: Estudo exploratório com o Marwit-Meuser Caregiver Grief Inventory (Short Form). Dissertação de Mestrado Integrado, Faculdade de Psicologia e de Ciências da Educação da Universidade de Coimbra, Portugal.. Acedido em https://estudogeral.sib.uc.pt/handle/10316/27807. 
Gilbert, K. (1996). "We've had the same loss, why don't we have the same grief?" Loss and differential grief in families. Death Studies, 20, 269-283.

Gjersing, L., Caplehorn, J., \& Clausen, T. (2010). Cross-cultural adaptation of research instruments: Language, setting, time and statistical considerations. BMC Medical Research Methodology, 10(13), 101-110. doi: 10.1186/1471-2288-10-13

Grassi, L. (2007). Bereavement in families with relatives dying of cancer. Current Opinion in Supportive and Palliative Care, 1, 43-49. doi: 10.1097/SPC.0b013e32813a3276

Guldin, M., Vedsted, P., Zachariae, F., Olesen, F., \& Jensen, A. (2012). Complicated grief and need for professional support in family caregivers of cancer patients in palliative care: A longitudinal cohort study. Supportive Care in Cancer, 20, 1679-1685. doi: 10.1007/ s00520-011-1260-3

Kissane, D., \& Bloch, S. (2002). Family focused grief therapy: A model of family-centered care during palliative care and bereavement. London: Open University Press.

Kissane, D., Bloch, S., Dowe, D., Snyder, R., Onghena, P., McKenzie, D., \& Wallace, C. (1996). The Melbourne family grief study, I: Perceptions of family functioning in bereavement. The American Journal of Psychiatry, 153(5), 650-658.

Lichtenthal, W., Prigerson, H., \& Kissane, D. (2010). Bereavement: A special issue in oncology. In J. Holland, W. Breitbart, P. Jacobsen, M. Lederberg, M. Loscalzo, \& R. McCorkle (Eds.). Psycho-Oncology (2nd ed., pp. 537-543). New York: Oxford University Press.

Lindemann, E. (1944). Symptomatology and management of acute grief. American Journal of Psychiatry, 101, 141-148.

Marôco, J. (2010). Análise de equações estruturais: Fundamentos teóricos, software \& aplicações. Pêro Pinheiro: ReportNumber.

Marwit, S., Chibanll, J., Dougherty, R., Jenkins, C., \& Shawgo, J. (2008). Assessing predeath grief in cancer caregivers using the Marwit-Meuser Caregiver Grief Inventory (MM-CGI). Psycho-Oncology, 17, 300-307. doi: 10.1002/pon.1218

Marwit, S., \& Kaye, P. (2006). Measuring grief in caregivers of persons with acquired brain injury, Brain Injury, 20, 1419-1429. doi: 10.1080/02699050601082214

Marwit, S., \& Meuser, T. (2002). Development and initial validation of an inventory to assess grief in caregiver's of persons with Alzheimer's disease. The Gerontologist, 42(6), 751-765.

Marwit, S., \& Meuser, T. (2005). Development of a short form inventory to assess grief in caregivers of dementia patients. Death Studies, 29, 191-205. doi: 10.1080/07481180590916335

Moos, N. (1995). An integrative model of grief. Death Studies, 19, 337-364.

Pestana, M. H., \& Gageiro, J. (2008). Análise de dados para ciências sociais - A complementaridade do SPSS ( $5^{\text {a }}$ ed.). Lisboa: Sílabo.

Reynolds, L., \& Botha, D. (2006). Anticipatory grief: Its nature, impact, and reasons for contradictory findings. Counselling, Psychotherapy, and Health, 2(2), 15-26.

Rolland, J. (1990). Anticipatory loss: A family systems developmental framework. Family Process, 29(3), 229-244.

Tercero, R. (2002). Duelo familiar. Sistemas Familiares, 18(1-2), 48-61.

Walsh, F., \& McGoldrick (2004). Loss and the family: A systemic perspective. In F. Walsh, \& M. McGoldrick (Eds). Living beyond the loss. Death in the family (2nd ed., pp. 3-26). New York: W. W. Norton \& Company.

Wedemeyer, N. (1986). Transformation of family images related to death. Journal of Family Issues, 7, 277-296.

Zilberfein, F. (1999). Coping with death: Anticipatory grief and bereavement. Generations, 23(1) 69-74. 